\title{
THE POTENTIAL KERNEL AND HITTING PROBABILITIES FOR THE GENERAL STABLE PROCESS IN $R^{N}$
}

\author{
BY \\ W. E. PRUITT( $\left.{ }^{1}\right)$ AND S. J. TAYLOR
}

1. Introduction. Let $X(t)$ be a stable process and $p(t, x)$ be the density of $X(t)$. For all the stable distributions of index $\alpha$ on the line, $|x|^{1+\alpha} p(1, x)$ is bounded [11]. This ensures that the hitting probabilities of spheres for these processes (as long as the processes are type $A$ ) are of the same order of magnitude as for the symmetric stable process of index $\alpha$. In higher dimensions the situation is more complicated, and there has been little study of the asymptotic behaviour of the density function except in the symmetric case and in the case of Brownian motion. There is no problem for Brownian motion since the density function is known so we may as well assume that $\alpha<2$. For the symmetric stable density of index $\alpha$ in $R^{N}$, $|x|^{N+\alpha} p(1, x)$ is bounded (and, in fact, tends to a limit as $\left.|x| \rightarrow \infty\right)$ [1]. To see that this is not the case in general consider the stable density of index $\alpha$ in $R^{2}$ given by

$$
p_{2}(1, x)=p_{1}\left(1, x_{1}\right) p_{1}\left(1, x_{2}\right)
$$

where $x=\left(x_{1}, x_{2}\right)$ and $p_{1}$ denotes the density of the symmetric stable process of index $\alpha$ in $R^{1}$. If we let $x$ tend to infinity along one of the axes, $p_{2}(1, x)$ decays like $|x|^{-1-\alpha}$, while if we go along any other ray from the origin it behaves like $|x|^{-2-2 \alpha}$. This example extends to $R^{N}$ and we shall prove that it illustrates the worst possible behaviour of the density $p(1, x)$ so that $|x|^{1+\alpha} p(1, x)$ is bounded for any stable process. (Our treatment of the stable processes of index one is restricted to those which satisfy the scaling property; see $\$ 2$.)

One of our objectives is to obtain conditions on the form of the stable distribution which will ensure a decay rate for the density closer to that for the symmetric process. In terms of the measure $\mu$ in the usual representation of the characteristic function (see $\$ 2$ ), we use a condition that $\mu$ is "not too concentrated" near any point to improve the estimate of the asymptotic behaviour. The form of this condition is fairly satisfactory for $N=2$, but it does not give enough information for $N \geqq 3$. It seems plausible that, at least when the defining measure $\mu$ has a bounded density on the unit sphere, $|x|^{N+\alpha} p(1, x)$ should be bounded. Our method of proof does not get us anywhere near this result for general $N$. However, it does imply

Received by the editors March 6, 1969.

( ${ }^{1}$ Research was supported in part by NSF Grant GP-7490.

Copyright (C) 1969, American Mathematical Society 
that for such a $\mu,|x|^{2+\alpha-\varepsilon} p(1, x)$ is bounded for every fixed $\varepsilon>0$, which is close to the conjecture when $N=2$. The proof of the main Theorem 1 , as given in $\S 3$, is rather involved as it requires the use of $N$-dimensional Fourier transforms.

It was pointed out to us by S. Port that the alleged proof of the estimate (16) in [12] is invalid. The example we have already considered-the convolution of symmetric 1-dimensional distributions of index $\alpha$ on each axis-shows that the kernel $U(x)$ may take the value $+\infty$ for some $x$ with $|x|=1$, if $\alpha \leqq(N-1) / 2$. It follows that Lemma 4 of [12] is false in general. Thus the hitting probabilities for spheres in dimension $N \geqq 2$ are not of the same order of magnitude for all type $A$ stable processes of index $\alpha$. The main object of $\$ 5$ of the present paper is to obtain valid estimates of these hitting probabilities. In order to do this, we first have to estimate the average value of $U(x)$ over a small neighbourhood; this is done in $\$ 4$.

There are other consequences of Theorem 1. For example, it shows that if $\alpha>(N-1) / 2$, then $U(x)$ is continuous on the unit sphere and it is then a consequence of the discussion in Port [8, p. 367] that his Theorem 2 is valid if the process is type $A$. This theorem concludes that the limit as the initial point tends to infinity of the conditional hitting distribution of a bounded Borel set exists and is the normalized cocapacitory measure of the set.

$\$ 6$ contains a detailed discussion of the example where $\mu$ consists of point masses. This discussion shows that the results of $\$ \S 3,4$, and 5 are best possible in general. An intuitive explanation of why the hitting probabilities are different for this example is also provided.

It is worth remarking that the inaccurate estimates of hitting probabilities in [12] do not invalidate the main results of that paper. The evaluation of the correct Hausdorff measure function for measuring the range of the process is accurate as one can use Lemma 6.1 of [10] to give an estimate of the number of cubes entered by the process. Furthermore, Theorems 2 and 3, stated without proof, are correct because it turns out that if we estimate the "delayed hitting probability" of a sphere, i.e. the probability of entering the sphere after unit time has elapsed, then this does have the same order of magnitude for all stable processes of type $A$ and index $\alpha$ in $R^{N}$. This estimate of the "delayed hitting probability" is given in $\S 7$. Some remaining problems are discussed in $§ 8$.

2. Preliminaries. The $N$-dimensional characteristic function of a stable process $X(t)$ of index $\alpha$ has the form $\exp [t \psi(\xi)]$ where

$$
\psi(\xi)=i(\xi, b)-c|\xi|^{\alpha} \int_{S_{N}} w_{\alpha}(\xi, \theta) \mu(d \theta)
$$

with $b \in R^{N}, c>0$,

$$
\begin{aligned}
& w_{\alpha}(\xi, \theta)=[1-i \operatorname{sgn}(\xi, \theta) \tan \pi \alpha / 2]|(\xi /|\xi|, \theta)|^{\alpha}, \quad \alpha \neq 1, \\
& w_{1}(\xi, \theta)=|(\xi /|\xi|, \theta)|+(2 i / \pi)(\xi /|\xi|, \theta) \log |(\xi, \theta)|,
\end{aligned}
$$


and $\mu$ is a probability measure on the surface of the unit sphere $S_{N}$ in $R^{N}$ [5]. We shall assume that $\mu$ is not supported by a proper subspace of $R^{N}$, and that $b=0, c=1$. The process is called the symmetric stable process of index $\alpha$ in $R^{N}$ when $\mu$ is uniform.

The density function $p(t, x)$ of $X(t)$ is continuous and bounded in $x$ for fixed $t$. It also satisfies the scaling property (except for some nonsymmetric processes of index 1)

$$
p(t, x)=p\left(r t, r^{1 / \alpha} x\right) r^{N / \alpha}
$$

for all $r>0$, or in terms of the process itself, $X(r t)$ and $r^{1 / \alpha} X(t)$ have the same distribution. The scaling property is satisfied when $\alpha=1$ if and only if the $\mu$ distribution satisfies

$$
\int_{S_{N}} \theta \mu(d \theta)=0
$$

We shall consider only those processes which satisfy (2.2) when $\alpha=1$. The stable processes have been classified in [12] as being of type $A$ if $p(1,0)>0$ and of type $B$ otherwise. When $\alpha \geqq 1$, only processes of type $A$ can occur.

We assume that our process satisfies the conditions of Hunt's hypothesis (A) [3]. Thus the sample functions $X(t)$ will be right continuous and have left limits everywhere. The process will also have the strong Markov property. Since we only need to consider $N \geqq 2$, and since there is no problem with planar Brownian motion we can and will assume $N>\alpha$. This allows us to use the ordinary potential kernel for the process. Since we have a continuous density $p(t, x)$, the kernel of the process has a density with respect to Lebesgue measure given by $u(x, y)=U(y-x)$ where

$$
U(x)=\int_{0}^{\infty} p(t, x) d t
$$

and Hunt's hypothesis (F) is also satisfied. This means that, at least for compact sets $E$ of positive natural capacity, there is a nonnull measure $\nu$, concentrated on $E$, such that

$$
\Phi(x, E)=P^{x}[X(t) \in E \text { for some } t>0]=\int_{E} u(x, y) \nu(d y) .
$$

The defining integral in (2.3) may diverge for some $x$, but it is well defined. We will see in $\S 4$ that (2.3) converges for a.e. $x$.

We shall use the notation

$$
g(\xi)=\int_{S_{N}} w_{\alpha}(\xi, \theta) \mu(d \theta)
$$

and note that for the processes being considered this is actually a function of $\xi /|\xi|$. Let the real valued function $a_{\alpha}$ be defined by

$$
\begin{aligned}
a_{\alpha}(y)=y^{\alpha}, & \text { if } y \geqq 0, \\
=0, & \text { if } y<0,
\end{aligned}
$$


and let

$$
\begin{aligned}
& g_{1}(\xi)=\int_{S_{N}} a_{\alpha}((\xi /|\xi|, \theta)) \mu(d \theta), \\
& g_{2}(\xi)=\int_{S_{N}} a_{\alpha}(-(\xi|| \xi \mid, \theta)) \mu(d \theta) .
\end{aligned}
$$

Then for $\alpha \neq 1$, we have

$$
g(\xi)=\sec (\pi \alpha / 2)\left\{g_{1}(\xi) e^{-i \pi \alpha / 2}+g_{2}(\xi) e^{i \pi \alpha / 2}\right\} .
$$

Furthermore

$$
\operatorname{Re} g(\xi)=g_{1}(\xi)+g_{2}(\xi)=\int_{S_{N}}|(\xi /|\xi|, \theta)|^{\alpha} \mu(d \theta) .
$$

Since we assumed that $\mu$ does not concentrate on a proper subspace of $S_{N}$, we have $\operatorname{Re} g(\xi)>0$, and since it is continuous $\operatorname{Re} g(\xi)$ is bounded below by a positive constant $\gamma$. (Recall that $g$ is actually a function on the compact $S_{N}$.) We also have $0 \leqq g_{1}(\xi) \leqq 1,0 \leqq g_{2}(\xi) \leqq 1$, so that all values of $g(\xi)$ are taken in the rectangular domain

$$
D=\{\sigma=u+i v: \gamma \leqq u \leqq 1,|v| \leqq \tan \pi \alpha / 2\}
$$

It is important to note that while the rectangle $D$ depends on $\mu$ through $\gamma, \gamma$ (and hence $D$ ) is invariant under orthogonal transformations on $\mu$. For $\alpha=1$, a similar analysis with (assuming (2.2)) $g(\xi)=g_{R}(\xi)+i g_{I}(\xi)$ where

$$
\begin{aligned}
g_{R}(\xi) & =\int_{S_{N}}|(\xi /|\xi|, \theta)| \mu(d \theta), \\
g_{I}(\xi) & =(2 / \pi) \int_{S_{N}}(\xi /|\xi|, \theta) \log |(\xi /|\xi|, \theta)| \mu(d \theta),
\end{aligned}
$$

shows that $g(\xi)$ takes its values in the rectangle

$$
D=\{\sigma=u+i v: \gamma \leqq u \leqq 1,|v| \leqq 2 / \pi e\} .
$$

The inversion formula allows us to write

$$
p(1, x)=(2 \pi)^{-N} \int_{R^{N}} \exp \left(-i(x, \xi)-|\xi|^{\alpha} g(\xi)\right) d \xi
$$

In estimating the asymptotic behaviour of the density, we are only interested (for the application to hitting probabilities) in letting $x$ tend to infinity along rays from the origin and we want bounds which are independent of the particular direction of approach. Therefore there will be no loss in assuming that the direction we take to infinity is along the positive first coordinate axis since this can be achieved in any case by an orthogonal transformation on $x$, which can be converted to one on $\mu$, and all the bounds obtained will be invariant under orthogonal 
transformations on $\mu$. We shall use the shorthand notation $\rho$ for $(\rho, 0,0, \ldots, 0)$; then

$$
p(1, \rho)=(2 \pi)^{-N} \int_{R^{N}} \exp \left(-i \rho \xi_{1}-|\xi|^{\alpha} g(\xi)\right) d \xi
$$

Making the usual change to spherical coordinates,

$$
\begin{aligned}
\xi_{j} & =r \sin \varphi_{1} \sin \varphi_{2} \ldots \sin \varphi_{j-1} \cos \varphi_{j}, \quad 1 \leqq j \leqq N-1, \\
\xi_{N} & =r \sin \varphi_{1} \sin \varphi_{2} \ldots \sin \varphi_{N-2} \sin \varphi_{N-1},
\end{aligned}
$$

where $0 \leqq r<\infty, 0 \leqq \varphi_{j} \leqq \pi$ for $1 \leqq j \leqq N-2,0 \leqq \varphi_{N-1} \leqq 2 \pi$, the Jacobian is

and

$$
J\left(r, \varphi_{1}, \ldots, \varphi_{N-1}\right)=r^{N-1}\left(\sin \varphi_{1}\right)^{N-2}, \ldots,\left(\sin \varphi_{N-3}\right)^{2} \sin \varphi_{N-2},
$$

$$
\begin{gathered}
p(1, \rho)=(2 \pi)^{-N} \int_{0}^{2 \pi} \cdots \int_{0}^{\pi} \int_{0}^{\infty} \exp \left(-\operatorname{ir} \rho \cos \varphi_{1}-r^{\alpha} h\left(\varphi_{1}, \ldots, \varphi_{N-1}\right)\right) \\
J\left(r, \varphi_{1}, \ldots, \varphi_{N-1}\right) d r d \varphi_{1} \ldots d \varphi_{N-1},
\end{gathered}
$$

where we have let

$$
h\left(\varphi_{1}, \ldots, \varphi_{N-1}\right)=g\left(r \cos \varphi_{1}, \ldots, r \sin \varphi_{1} \sin \varphi_{2} \ldots \sin \varphi_{N-1}\right) .
$$

This is the formula which will prove most useful in estimating the asymptotic behaviour of $p(1, x)$.

We will use $M, c, K$ to denote finite positive constants whose precise values are unimportant; these may differ when the same symbol occurs in distinct contexts.

3. Asymptotic bounds for the stable density. Our main aim is to give a proof of

THEOREM 1. Suppose that for some $\beta \geqq 0$, there is an $M$ such that

$$
\int_{S_{N}}|(\xi, \theta)|^{-\beta} \mu(d \theta) \leqq M
$$

for all $\xi \in S_{N}$. Then there is a constant $K$ such that $p(1, x) \leqq K|x|^{-\alpha-\beta-1}$.

COROLlaRY. For any stable distribution of index $\alpha$ (satisfying (2.2) if $\alpha=1$ ) in $R^{N}$, $|x|^{1+\alpha} p(1, x)$ is bounded.

The corollary follows immediately from the theorem since the hypothesis is trivially satisfied for $\beta=0$. Unfortunately the improvement in the bound on the rate of decay given by the theorem is limited since the hypothesis can only be satisfied if $\beta<1$. In the planar case, this is not too important as we believe the result is close to best possible. We will see later what would be necessary in order to sharpen the method of proof to yield better results for $N \geqq 3$. The proof of the theorem requires heavy computation so we break it into a sequence of lemmas.

The first lemma establishes a result about the order of magnitude of an integral involving Bessel functions. We give the proof because we have been unable to find 
the exact result in the literature. However, the method of proof is that used to obtain the asymptotic behaviour of the symmetric stable density by Polya [7] in $R^{1}$ and Blumenthal and Getoor [1] in $R^{N}, N \geqq 2$. The notation and integration formulas for Bessel functions can be found in [2] or [13].

LEMma 1. Let $J_{v}(y)$ denote the Bessel function of the first kind and let $\sigma \in D$. Then if $\rho>0, \nu \geqq 0, \beta+\nu>-1$,

$$
\begin{aligned}
\int_{0}^{\infty} J_{\nu}(r \rho) \exp \left(-\sigma r^{\alpha}\right) r^{\beta} d r & =O\left(\rho^{-\alpha-\beta-1}\right), & & \text { if } \beta=\nu+1, \\
& =O\left(\rho^{-\beta-1}\right), & & \text { if } \beta \neq \nu+1,
\end{aligned}
$$

the constants being uniform for $\sigma \in D$.

Proof. An integration by parts and change of variable yield

$$
\begin{aligned}
\int_{0}^{\infty} J_{v}(r \rho) \exp \left(-\sigma r^{\alpha}\right) r^{\beta} d r= & \rho^{-\alpha-\beta-1} \int_{0}^{\infty} J_{v+1}(s) \exp \left(-\sigma(s / \rho)^{\alpha}\right) \sigma \alpha s^{\alpha+\beta-1} d s \\
& -(\beta-v-1) \rho^{-\beta-1} \int_{0}^{\infty} J_{v+1}(s) \exp \left(-\sigma(s / \rho)^{\alpha}\right) s^{\beta-1} d s
\end{aligned}
$$

Thus when $\beta=\nu+1$, it only remains to show that the first integral on the right is bounded as $\rho \rightarrow \infty$. In order to do this, we introduce the Bessel function of the third kind $H_{v+1}^{(1)}(s)=J_{v+1}(s)+i Y_{v+1}(s)$ and work separately with the real and imaginary parts of the integral. Letting $\sigma=u+i v$,

$$
\begin{aligned}
\operatorname{Re} & \int_{0}^{\infty} J_{v+1}(s) \exp \left(-\sigma(s / \rho)^{\alpha}\right) \sigma \alpha s^{\alpha+\beta-1} d s \\
& =\int_{0}^{\infty} J_{v+1}(s) \exp \left(-u(s / \rho)^{\alpha}\right) \alpha\left\{u \cos \left[v(s / \rho)^{\alpha}\right]+v \sin \left[v(s / \rho)^{\alpha}\right]\right\} s^{\alpha+\beta-1} d s \\
& =\operatorname{Re} \int_{0}^{\infty} H_{v+1}^{(1)}(s) \exp \left(-u(s / \rho)^{\alpha}\right) \alpha\left\{u \cos \left[v(s / \rho)^{\alpha}\right]+v \sin \left[v(s / \rho)^{\alpha}\right]\right\} s^{\alpha+\beta-1} d s .
\end{aligned}
$$

Now choose a small positive angle $\varphi_{0}$ so that

$$
\gamma \cos \alpha \varphi-\tan (\pi \alpha / 2) \sin \alpha \varphi>0
$$

for all $\varphi \in\left[0, \varphi_{0}\right]$. (If $\alpha=1, \tan \pi \alpha / 2$ should be replaced by $2 / \pi e$.) This choice of $\varphi_{0}$ ensures that

$$
u \cos \alpha \varphi-|v| \sin \alpha \varphi>0
$$

for all $\varphi \in\left[0, \varphi_{0}\right]$ and $\sigma \in D$. By considering the usual contour integral, we see that we can change the integral to one along the ray $s \exp \left\{i \varphi_{0}\right\}, s \geqq 0$. This gives the Bessel function a negative exponential behaviour so that the passage to the limit may be carried out. The imaginary part of the integral can be handled in exactly the same way.

For the other estimate (when $\beta \neq \nu+1$ ), we start with a change of variable:

$$
\int_{0}^{\infty} J_{v}(r \rho) \exp \left(-\sigma r^{\alpha}\right) r^{\beta} d r=\rho^{-\beta-1} \int_{0}^{\infty} J_{v}(s) \exp \left(-\sigma(s / \rho)^{\alpha}\right) s^{\beta} d s
$$


Again we must show that the integral is bounded, but if we proceed as before there are integrability difficulties near zero when $\beta \leqq \nu-1$. So we first observe that the integral from zero to one is bounded, independent of $\rho$ and $\sigma$, since $\left|J_{v}(s)\right| \leqq c s^{\nu}$. To handle the integral from one to infinity, we introduce $H_{v}^{(1)}(s)$ and work separately with the real and imaginary parts of the integral as before. With the same $\varphi_{0}$, we now use the contour made up of a segment of the real axis, an arc of the circle $|z|=R$, a segment of the ray from the origin through $\exp \left\{i \varphi_{0}\right\}$, and an arc of the unit circle. The integral along the large arc and along the ray in the $\varphi_{0}$ direction are bounded as before, while it is easy to see that the integral along the arc of the unit circle is also bounded, independent of $\rho$ and $\sigma$.

We now want to establish estimates (contained in Lemmas 2, 3, and 4) for integrals which will occur when we invert the characteristic function to obtain the stable density. The proof of Lemma 2 is very similar to that of Lemma 1, while the other two are easy consequences of Lemma 1.

LeMmA 2. Let $\sigma \in D$ and $\beta \geqq 0$. Then

$$
\int_{0}^{\infty} \exp (-i r y) \exp \left(-\sigma r^{\alpha}\right) r^{\beta} d r=O\left(|y|^{-\beta-1}\right)
$$

the constant being uniform for $\sigma \in D$.

Proof. If $y<0$, make the change of variable $s=-r y$ and then use the same contour used in the proof of the first part of Lemma 1 to change the integral to the ray in the $\varphi_{0}$ direction. If $y>0$, the change of variable is $s=r y$, and then the angle $\varphi_{0}$ should be the negative of the previous one.

Lemma 3. Let $\sigma \in D$ and $\rho>0$. Then

$$
\int_{0}^{\infty} \int_{0}^{\pi} \exp \left(-i r \rho \cos \varphi-\sigma r^{\alpha}\right) r^{N-1}(\sin \varphi)^{N-2} d \varphi d r=O\left(\rho^{-\alpha-N}\right)
$$

the constant being independent of $\sigma$.

Proof. Since

$$
\int_{0}^{\pi} \exp (-i r \rho \cos \varphi)(\sin \varphi)^{N-2} d \varphi=c J_{(N-2) / 2}(r \rho)(r \rho)^{-(N-2) / 2}
$$

this is an immediate consequence of Lemma 1.

LEMMA 4. Let $m, n$ be nonnegative integers and $\sigma \in D$. Then if $\beta \geqq 0, \rho>0$,

$$
\int_{0}^{\infty} \int_{0}^{\pi} \exp \left(-i r \rho \cos \varphi-\sigma r^{\alpha}\right) \sin ^{m} \varphi \cos ^{n} \varphi r^{\beta} d \varphi d r=O\left(\rho^{-\beta-1}\right),
$$

the constant being independent of $\sigma$. 
Proof. By use of the identity $\cos ^{2} \varphi=1-\sin ^{2} \varphi$, it suffices to consider $n=0,1$. Then the formulas

$$
\begin{gathered}
\int_{0}^{\pi} \exp (-i r \rho \cos \varphi) \sin ^{m} \varphi d \varphi=c J_{m / 2}(r \rho)(r \rho)^{-m / 2}, \\
\int_{0}^{\pi} \exp (-i r \rho \cos \varphi) \sin ^{m} \varphi \cos \varphi d \varphi=c J_{(m+2) / 2}(r \rho)(r \rho)^{-m / 2}
\end{gathered}
$$

together with Lemma 1 complete the proof.

To estimate the integral involved in inverting the characteristic function, we shall use a finite number of terms from a power series expansion in the integrand. The next three lemmas establish the estimates we need for this.

LeMmA 5. Let $\sigma_{1}, \sigma_{2} \in D, \rho>0$. Then

$$
\begin{aligned}
\int_{0}^{\infty} \exp (-i r \rho \cos \varphi)\left(\exp \left(-\sigma_{2} r^{\alpha}\right)-\exp \left(-\sigma_{1} r^{\alpha}\right) \sum_{j=0}^{m-1} \frac{\left(\sigma_{1}-\sigma_{2}\right)^{j}}{j !} r^{j \alpha}\right) r^{N-1} d r \\
=\left\{\begin{array}{l}
O\left(\left|\sigma_{2}-\sigma_{1}\right|^{m}\right) \\
O\left(\{\rho|\cos \varphi|\}^{-N-m \alpha}\left|\sigma_{2}-\sigma_{1}\right|^{m}\right) .
\end{array}\right.
\end{aligned}
$$

Proof. The first bound follows easily from the observation that

$$
\begin{aligned}
\exp \left(-\sigma_{2} r^{\alpha}\right)-\exp & \left(-\sigma_{1} r^{\alpha}\right) \sum_{j=0}^{m-1} \frac{\left(\sigma_{1}-\sigma_{2}\right)^{j}}{j !} r^{j \alpha} \\
= & (-1)^{m} \int_{\sigma_{1}}^{\sigma_{2}} \int_{\sigma_{1}}^{z_{m}} \ldots \int_{\sigma_{1}}^{z_{2}} \exp \left(-z_{1} r^{\alpha}\right) r^{m \alpha} d z_{1} \ldots d z_{m},
\end{aligned}
$$

and then using integration contours on the straight line from $\sigma_{1}$ to $\sigma_{2}$ which has the effect of keeping the contours in $D$ and also of making intermediate distances smaller. Thus

$$
\left|\exp \left(-\sigma_{2} r^{\alpha}\right)-\exp \left(-\sigma_{1} r^{\alpha}\right) \sum_{j=0}^{m-1} \frac{\left(\sigma_{1}-\sigma_{2}\right)^{j}}{j !} r^{j \alpha}\right| \leqq \exp \left(-\gamma r^{\alpha}\right) r^{m \alpha}\left|\sigma_{2}-\sigma_{1}\right|^{m}
$$

and this suffices for the first bound. Now, if we substitute in the integral we are estimating the right-hand side of (3.1) and do the $r$-integration first, we have the integral equal to

$$
(-1)^{m} \int_{\sigma_{1}}^{\sigma_{2}} \int_{\sigma_{1}}^{z_{m}} \cdots \int_{\sigma_{1}}^{z_{2}} \int_{0}^{\infty} \exp \left(-i r \rho \cos \varphi-z_{1} r^{\alpha}\right) r^{m \alpha+N-1} d r d z_{1} \ldots d z_{m} .
$$

Applying Lemma 2 to this expression yields the second bound.

LEMMA 6. Suppose $\alpha>0$ and $\alpha$ is not an integer, $\beta \geqq 0$, and $k$ is chosen so that

$$
k-1<\alpha+\beta \leqq k .
$$


Then

$$
\left|a_{\alpha}(v)-\sum_{j=0}^{k-1} a_{\alpha-j}(u) \frac{(\alpha)_{j}}{j !}(v-u)^{j}\right|(\min \{|u|,|v|\})^{\beta}=O\left(|v-u|^{\alpha+\beta}\right) .
$$

(Here $(\alpha)_{j}=\alpha(\alpha-1) \ldots(\alpha-j+1)$, and $a_{\alpha}$ is defined in (2.6).)

Proof. We give the proof only for $|v| \leqq|u|$, the two cases being essentially the same. First suppose $0 \leqq v \leqq u$; then

$$
\begin{aligned}
\left|v^{\alpha}-\sum_{j=0}^{k-1} u^{\alpha-j} \frac{(\alpha)_{j}}{j !}(v-u)^{j}\right| v^{\beta} & =v^{\beta} \int_{v}^{u} \int_{y_{k}}^{u} \ldots \int_{y_{2}}^{u} y_{1}^{\alpha-k}\left|(\alpha)_{k}\right| d y_{1} \ldots d y_{k} \\
& \leqq \int_{v}^{u} \int_{y_{k}}^{u} \ldots \int_{y_{2}}^{u} y_{1}^{\alpha+\beta-k}\left|(\alpha)_{k}\right| d y_{1} \ldots d y_{k} \\
& \leqq \int_{v}^{u} \int_{y_{k}}^{u} \ldots \int_{0}^{u-y_{2}} y_{1}^{\alpha+\beta-k}\left|(\alpha)_{k}\right| d y_{1} \ldots d y_{k} \\
& =\frac{\left|(\alpha)_{k}\right|}{(\alpha+\beta)_{k}}(u-v)^{\alpha+\beta} .
\end{aligned}
$$

Next, consider the case $0 \leqq-v \leqq u$. Then we must show that

$$
\left\{\sum_{j=0}^{k-1} u^{\alpha-j} \frac{(\alpha)_{j}}{j !}(u-v)^{j}\right\}|v|^{\beta}=O\left((u-v)^{\alpha+\beta}\right) .
$$

In this case, the bound is valid for each individual term, i.e.

$$
u^{\alpha-j}(u-v)^{j}|v|^{\beta} \leqq u^{\alpha+\beta-j}(u-v)^{j} \leqq(u-v)^{\alpha+\beta}
$$

since $u \leqq u-v$ and $\alpha+\beta-j>0$. Finally, if $u<0$, the bound is trivial.

Next we give the lemma which will take the place of Lemma 6 when $\alpha=1$. In this case, $g_{R}(\xi)$ is easy to deal with, but $g_{I}(\xi)$ is complicated because of the log term. In order to get rid of the log term, we use symmetric second differences. To make the later work easier we will also use second differences for the real part. The first part of the lemma is valid for all $\beta$, and the second part could be made to work for $\beta>1$ by subtracting more terms in the series expansion. We restrict our attention to $\beta \leqq 1$ since that is the only case that can be used.

Lemma 7. Suppose $0 \leqq \beta \leqq 1$. Then

$$
\begin{gathered}
|| u+v|+| u-v|-2| u||(\min \{|u|,|u+v|,|u-v|\})^{\beta}=O\left(|v|^{1+\beta}\right) \\
|(u+v) \log | u+v|+(u-v) \log | u-v|-2 u \log | u||(\min \{|u|,|u+v|,|u-v|\})^{\beta} \\
=O\left(|v|^{1+\beta}\right) .
\end{gathered}
$$

Proof. It clearly suffices to consider $v>0$. The first statement is trivial. To prove the second, note that

$$
(u+v) \log |u+v|+(u-v) \log |u-v|-2 u \log |u|=\int_{0}^{v} \log \left|\frac{u+z}{u-z}\right| d z .
$$


The integrand is nonnegative for $u \geqq 0$ and is an odd function of $u$. We will therefore consider only $u \geqq 0$ and consider only upper bounds for the integral. If $u \geqq 2 v$, then

and since

$$
\int_{0}^{v} \log \left|\frac{u+z}{u-z}\right| d z \leqq \int_{0}^{v}\left(\frac{u+z}{u-z}-1\right) d z \leqq \frac{v^{2}}{u-v},
$$

$$
\mid\left(\left.\min \{|u|,|u+v|,|u-v|\}\right|^{\beta} \leqq(u-v)^{\beta},\right.
$$

combining these estimates gives the desired bound in this case. If $v \leqq u \leqq 2 v$,

$$
\int_{0}^{v} \log \left|\frac{u+z}{u-z}\right| d z \leqq \int_{0}^{u} \log \left|\frac{u+z}{u-z}\right| d z=\int_{1}^{\infty} \log y \frac{2 u}{(y+1)^{2}} d y \leqq c u \leqq 2 c v,
$$

and in this case we use the $u$ term from the minimum function to get the final bound. Finally, if $0 \leqq u \leqq v$,

$$
\begin{aligned}
\int_{0}^{v} \log \left|\frac{u+z}{u-z}\right| d z & =\int_{0}^{u} \log \left|\frac{u+z}{u-z}\right| d z+\int_{u}^{v} \log \left|\frac{u+z}{u-z}\right| d z \\
& \leqq c u+\int_{(u+v) /(v-u)}^{\infty} \log y \frac{2 u}{(y-1)^{2}} d y \\
& \leqq c u+(v-u)+\int_{2}^{\infty} \log y \frac{2 u}{(y-1)^{2}} d y \leqq c_{1} v
\end{aligned}
$$

and the $u$ term can again be used from the minimum function.

Now we are ready to apply Lemmas 6 and 7 to obtain estimates on the function $g$ defined by (2.5). For $\alpha \neq 1$, we need to introduce some new functions. Let

and

$$
\begin{aligned}
& g_{1 j}(\xi, \eta)=\frac{(\alpha)_{j}}{j !} \int_{S_{N}} a_{\alpha-j}((\xi, \theta))(\eta-\xi, \theta)^{j} \mu(d \theta), \\
& g_{2 j}(\xi, \eta)=\frac{(\alpha)_{j}}{j !} \int_{S_{N}} a_{\alpha-j}(-(\xi, \theta))(\xi-\eta, \theta)^{j} \mu(d \theta),
\end{aligned}
$$

$$
g_{j}(\xi, \eta)=\sec (\pi \alpha / 2)\left\{g_{1 j}(\xi, \eta) e^{-i \pi \alpha / 2}+g_{2 j}(\xi, \eta) e^{i \pi \alpha / 2}\right\},
$$

where we are assuming for the moment that the defining integrals converge. Then we have

LEMMA 8. Suppose $\alpha \neq 1$, and that for some $\beta \geqq 0$ there is an $M$ such that

$$
\int_{S_{N}}|(\xi, \theta)|^{-\beta} \mu(d \theta) \leqq M
$$

for all $\xi \in S_{N}$. Then, choosing $k$ as in (3.2), we have for all $\xi, \eta \in S_{N}$

$$
g(\eta)-g(\xi)-\sum_{j=1}^{k-1} g_{j}(\xi, \eta)=O\left(|\xi-\eta|^{\alpha+\beta}\right)
$$


Proof. First note that the defining integrals for $g_{j}(\xi, \eta)$ converge due to the assumption (3.3). Now apply Lemma 6 with $v=(\eta, \theta), u=(\xi, \theta)$ :

$$
\begin{aligned}
\mid a_{\alpha}((\eta, \theta))-\sum_{j=0}^{k-1} a_{\alpha-j}((\xi, \theta)) \frac{(\alpha)_{j}}{j !} & (\eta-\xi, \theta)^{j} \mid \\
& \leqq K|(\eta-\xi, \theta)|^{\alpha+\beta}\left\{|(\eta, \theta)|^{-\beta}+|(\xi, \theta)|^{-\beta}\right\} .
\end{aligned}
$$

Integrating with respect to $\mu$ over $S_{N}$ and using $|(\eta-\xi, \theta)| \leqq|\eta-\xi|$, we obtain

$$
\left|g_{1}(\eta)-g_{1}(\xi)-\sum_{j=1}^{k-1} g_{1 j}(\xi, \eta)\right| \leqq 2 M K|\eta-\xi|^{\alpha+\beta} .
$$

The same technique works for $g_{2}$ and the estimates for $g$ follow.

COROLlaRY. For $\xi, \eta \in S_{N}$,

$$
\begin{aligned}
g(\eta)-g(\xi) & =O\left(|\xi-\eta|^{\alpha}\right), & & \text { if } \alpha<1, \\
& =O(|\xi-\eta|), & & \text { if } \alpha>1 .
\end{aligned}
$$

Proof. Since (3.3) is always valid with $\beta=0$, the bound for $\alpha<1$ is an immediate consequence of the lemma. For $\alpha>1$, we have

$$
g(\eta)-g(\xi)-g_{1}(\xi, \eta)=O\left(|\xi-\eta|^{\alpha}\right),
$$

and it is clear that

$$
\left|g_{1}(\xi, \eta)\right| \leqq \alpha|\sec \pi \alpha / 2||\xi-\eta|
$$

for $\alpha>1$.

Before proving the sharp estimate that we need for $\alpha=1$, we shall consider what we could get by using the same technique used for $\alpha \neq 1$. The result corresponding to the corollary is not valid, but since $|\log | x||$ is dominated by $|x|^{-\varepsilon}$ for small $|x|$ for any $\varepsilon>0$, one can prove very much as above that for $\alpha=1$ and any fixed $\varepsilon>0$,

$$
g(\eta)-g(\xi)=O\left(|\xi-\eta|^{1-8}\right) .
$$

In fact, the estimate can even be improved to $O(|(\xi-\eta) \log | \xi-\eta||)$, but (3.4) is good enough for our purposes. However, we do need an improved estimate involving the symmetric second difference of $g$. This is the subject of

LEMMA 9. Suppose $\alpha=1$, and that for some $0 \leqq \beta \leqq 1$ there is an $M$ such that (3.3) holds for all $\xi \in S_{N}$. Then

$$
g(\xi+\zeta)+g(\xi-\zeta)-2 g(\xi)=O\left(|\zeta|^{1+\beta}\right)
$$

whenever $\xi \in S_{N},|\xi+\zeta| \geqq 1,|\xi-\zeta| \geqq 1$.

Proof. We apply Lemma 7 with $u=(\xi, \theta), v=(\zeta, \theta)$ to obtain

$$
\begin{aligned}
||(\xi+\zeta, \theta)|+|(\xi-\zeta, \theta)|-2|(\xi, \theta)|| \\
\quad \leqq K|(\zeta, \theta)|^{1+\beta}\left\{|(\xi, \theta)|^{-\beta}+|(\xi+\zeta, \theta)|^{-\beta}+|(\xi-\zeta, \theta)|^{-\beta}\right\} \\
\quad \leqq K|\zeta|^{1+\beta}\left\{|(\xi, \theta)|^{-\beta}+|(\xi+\zeta|| \xi+\zeta \mid, \theta)|^{-\beta}+|(\xi-\zeta /|\xi-\zeta|, \theta)|^{-\beta}\right\},
\end{aligned}
$$


the last inequality being a consequence of the fact that $|\xi+\zeta| \geqq 1,|\xi-\zeta| \geqq 1$. Integrating with respect to $\mu$ and using (3.3), we get

$$
|\xi+\zeta| g_{R}(\xi+\zeta)+|\xi-\zeta| g_{R}(\xi-\zeta)-2 g_{R}(\xi)=O\left(|\zeta|^{1+\beta}\right)
$$

Now by the parallelogram law,

and thus

$$
2+2|\zeta|^{2}=|\xi+\zeta|^{2}+|\xi-\zeta|^{2} \geqq 1+|\xi-\zeta|^{2}
$$

$$
\begin{aligned}
\left|(|\xi-\zeta|-1) g_{R}(\xi-\zeta)\right| & \leqq \frac{|\xi-\zeta|^{2}-1}{|\xi-\zeta|+1} \leqq \frac{2|\zeta|^{2}}{|\xi-\zeta|^{2}} \leqq \min \left\{2|\zeta|,|\zeta|^{2}\right\} \\
& \leqq 2|\zeta|^{1+\beta} .
\end{aligned}
$$

In the same way we see that $\left|(|\xi+\zeta|-1) g_{R}(\xi+\zeta)\right| \leqq 2|\zeta|^{1+\beta}$. The imaginary part of $g$ can be estimated in exactly the same way by using the other half of Lemma 7 and the fact that

$$
(\xi+\zeta, \theta) \log |(\xi+\zeta /|\xi+\zeta|, \theta)|=(\xi+\zeta, \theta) \log |(\xi+\zeta, \theta)|-(\xi+\zeta, \theta) \log |\xi+\zeta|
$$

so that when the $\theta$ integration is performed the last term is zero because of the assumption (2.2). Thus we have that both the real and imaginary parts of $g$ satisfy the conclusion of the lemma.

These estimates on $g$ convert directly to bounds on the function $h$ defined in (2.9). The situation which will interest us is when

and

$$
\begin{aligned}
\xi_{1} & =0, \\
\xi_{j} & =\sin \varphi_{2} \ldots \sin \varphi_{j-1} \cos \varphi_{j}, \quad 2 \leqq j \leqq N-1, \\
\xi_{N} & =\sin \varphi_{2} \ldots \sin \varphi_{N-2} \sin \varphi_{N-1},
\end{aligned}
$$

$$
\begin{aligned}
\eta_{1} & =\cos \varphi_{1}, \\
\eta_{j} & =\sin \varphi_{1} \ldots \sin \varphi_{j-1} \cos \varphi_{j}, \quad 2 \leqq j \leqq N-1, \\
\eta_{N} & =\sin \varphi_{1} \ldots \sin \varphi_{N-2} \sin \varphi_{N-1} .
\end{aligned}
$$

For this particular case, it is useful to note that $|\xi-\eta| \leqq\left|\varphi_{1}-\pi / 2\right|$. Then, for example, we have from the corollary to Lemma 8 that

$$
\begin{aligned}
h\left(\varphi_{1}, \varphi_{2}, \ldots, \varphi_{N-1}\right)-h\left(\pi / 2, \varphi_{2}, \ldots, \varphi_{N-1}\right) & =O\left(\left|\varphi_{1}-\frac{\pi}{2}\right|^{\alpha}\right), \quad \text { if } \alpha<1, \\
& =O\left(\left|\varphi_{1}-\frac{\pi}{2}\right|\right), \quad \text { if } \alpha>1,
\end{aligned}
$$

the bounds being uniform in $\varphi_{2}, \ldots, \varphi_{N-1}$. For the case of $\alpha=1$, we have

COROLlARY. If $\alpha=1,0 \leqq \beta \leqq 1$, and there is an $M$ such that (3.3) holds for all $\xi \in S_{N}$, then

$$
\begin{aligned}
h\left(\varphi_{1}, \varphi_{2}, \ldots, \varphi_{N-1}\right)+h\left(\pi-\varphi_{1}, \varphi_{2}, \ldots, \varphi_{N-1}\right)-2 h(\pi / 2, & \left.\varphi_{2}, \ldots, \varphi_{N-1}\right) \\
& =O\left(\left|\varphi_{1}-\pi / 2\right|^{1+\beta}\right) .
\end{aligned}
$$


Proof. Let $\xi$ be as in (3.5) and $\zeta=\left(\operatorname{ctn} \varphi_{1}, 0, \ldots, 0\right)$. Then $|\xi+\zeta|=\left(1+\operatorname{ctn}^{2} \varphi_{1}\right)^{1 / 2}$ $=\left(\sin \varphi_{1}\right)^{-1}$ and we have $(\xi+\zeta)|\xi+\zeta|^{-1}=\eta$ as given in (3.6). Thus

$$
g(\xi+\zeta)=g\left((\xi+\zeta)|\xi+\zeta|^{-1}\right)=h\left(\varphi_{1}, \varphi_{2}, \ldots, \varphi_{N-1}\right)
$$

and in a similar way

$$
g(\xi-\zeta)=h\left(\pi-\varphi_{1}, \varphi_{2}, \ldots, \varphi_{N-1}\right) .
$$

Lemma 9 now yields the corollary for $\varphi_{1} \in[\pi / 4,3 \pi / 4]$, say; there is no problem for $\varphi_{1}$ outside this interval since $h$ is bounded.

Proof of Theorem 1. We consider first the situation when $\alpha \neq 1$. Recalling (2.8), let

$$
I(\rho)=\int_{0}^{\infty} \int_{0}^{\pi} \exp \left(-i r \rho \cos \varphi_{1}-r^{\alpha} h\left(\varphi_{1}\right)\right) r^{N-1}\left(\sin \varphi_{1}\right)^{N-2} d \varphi_{1} d r
$$

where the arguments $\varphi_{2}, \ldots, \varphi_{N-1}$ have been suppressed in the function $h$. In order to prove the theorem, it will suffice to show this integral is $O\left(\rho^{-\alpha-\beta-1}\right)$ uniformly in $\varphi_{2}, \ldots, \varphi_{N-1}$. First choose $m$ so that

$$
\begin{aligned}
m \geqq(N-1+\alpha) / \alpha, & \text { if } \alpha<1, \\
\geqq N-1+\alpha, & \text { if } \alpha>1 .
\end{aligned}
$$

Then let

$$
\begin{aligned}
& A_{1}\left(r, \varphi_{1}\right)=\exp \left(-r^{\alpha} h(\pi / 2)\right) \sum_{j=1}^{m-1} \frac{\left\{h(\pi / 2)-h\left(\varphi_{1}\right)\right\}^{j}}{j !} r^{j \alpha}, \\
& A_{2}\left(r, \varphi_{1}\right)=\exp \left(-r^{\alpha} h(\pi / 2)\right), \\
& A_{3}\left(r, \varphi_{1}\right)=\exp \left(-r^{\alpha} h\left(\varphi_{1}\right)\right)-A_{1}\left(r, \varphi_{1}\right)-A_{2}\left(r, \varphi_{1}\right),
\end{aligned}
$$

and

$$
I_{n}(\rho)=\int_{0}^{\infty} \int_{0}^{\pi} \exp \left(-i r \rho \cos \varphi_{1}\right) A_{n}\left(r, \varphi_{1}\right) r^{N-1}\left(\sin \varphi_{1}\right)^{N-2} d \varphi_{1} d r
$$

for $n=1,2,3$, the remaining $\varphi$ arguments still being suppressed. Now

$$
I(\rho)=I_{1}(\rho)+I_{2}(\rho)+I_{3}(\rho),
$$

and

$$
I_{2}(\rho)=O\left(\rho^{-\alpha-N}\right)
$$

by Lemma 3. If $\alpha<1$, by Lemma 5 and (3.7)

$$
\begin{aligned}
I_{3}(\rho) \leqq & K \int_{\left|\varphi_{1}-\pi / 2\right| \leqq \rho^{-1}}\left|h\left(\varphi_{1}\right)-h(\pi / 2)\right|^{m} d \varphi_{1} \\
& +K \int_{\left|\varphi_{1}-\pi / 2\right|>\rho^{-1}}\left|\rho \cos \varphi_{1}\right|^{-N-m \alpha}\left|h\left(\varphi_{1}\right)-h(\pi / 2)\right|^{m} d \varphi_{1} \\
\leqq & c \rho^{-m \alpha-1}+c \rho^{-m \alpha-1} \leqq c^{\prime} \rho^{-N-\alpha} .
\end{aligned}
$$


In the same way, one shows that if $\alpha>1$,

$$
I_{3}(\rho)=O\left(\rho^{-\alpha-N}\right) .
$$

To find suitable estimates for $I_{1}(\rho)$ we must expand $h\left(\varphi_{1}\right)-h(\pi / 2)$ to $k-1$ terms where $k$ is determined by (3.2). Define $h_{n}\left(\varphi_{1}\right)=g_{n}(\xi, \eta)$, where $\xi$ and $\eta$ are as in (3.5) and (3.6). Then by Lemma 8

$$
h\left(\varphi_{1}\right)-h(\pi / 2)-\sum_{n=1}^{k-1} h_{n}\left(\varphi_{1}\right)=O\left(\left|\varphi_{1}-\pi / 2\right|^{\alpha+\beta}\right) .
$$

Furthermore,

$$
\begin{aligned}
\left\{h(\pi / 2)-h\left(\varphi_{1}\right)\right\}^{j}-\left\{-\sum_{n=1}^{k-1} h_{n}\left(\varphi_{1}\right)\right\}^{j} & \\
= & \left\{h(\pi / 2)-h\left(\varphi_{1}\right)+\sum_{n=1}^{k-1} h_{n}\left(\varphi_{1}\right)\right\}_{\nu=0}^{j-1}\left\{h(\pi / 2)-h\left(\varphi_{1}\right)\right\}^{v}\left\{-\sum_{n=1}^{k-1} h_{n}\left(\varphi_{1}\right)\right\}^{j-1-\nu} \\
= & O\left(\left|\varphi_{1}-\pi / 2\right|^{\alpha+\beta}\right)
\end{aligned}
$$

for $j \leqq m-1$, since $m$ and $k$ depend only on $\alpha, \beta$, and $N$, and $h, h_{n}$ are bounded. Thus we define

$$
A_{4}\left(r, \varphi_{1}\right)=\exp \left(-r^{\alpha} h(\pi / 2)\right) \sum_{j=1}^{m-1}\left(\left\{-\sum_{n=1}^{k-1} h_{n}\left(\varphi_{1}\right)\right\}^{j} / j !\right) r^{j \alpha}
$$

and $I_{4}(\rho)$ by (3.8). We have shown that

$$
A_{1}\left(r, \varphi_{1}\right)-A_{4}\left(r, \varphi_{1}\right)=O\left(\left|\varphi_{1}-\pi / 2\right|^{\alpha+\beta} \exp \left(-\gamma r^{\alpha}\right) \sum_{j=1}^{m-1} r^{j \alpha}\right),
$$

and it follows that

$$
\int_{0}^{\infty} \exp \left(-\operatorname{ir} \rho \cos \varphi_{1}\right)\left\{A_{1}\left(r, \varphi_{1}\right)-A_{4}\left(r, \varphi_{1}\right)\right\} r^{N-1} d r=O\left(\left|\varphi_{1}-\pi / 2\right|^{\alpha+\beta}\right) .
$$

By applying Lemma 2 to the difference of the $j$ th terms in the sum, we find that for large values of $\rho\left|\cos \varphi_{1}\right|(3.12)$ is also

$$
O\left(\left|\rho \cos \varphi_{1}\right|^{-N-\alpha}\left|\varphi_{1}-\pi / 2\right|^{\alpha+\beta}\right) .
$$

Performing the $\varphi_{1}$ integration yields

$$
\begin{aligned}
\left|I_{1}(\rho)-I_{4}(\rho)\right| \leqq & K \int_{\left|\varphi_{1}-\pi / 2\right| \leqq \rho^{-1}}\left|\varphi_{1}-\pi / 2\right|^{\alpha+\beta} d \varphi_{1} \\
& +K \int_{\left|\varphi_{1}-\pi / 2\right|>\rho^{-1}}\left|\rho \cos \varphi_{1}\right|^{-N-\alpha}\left|\varphi_{1}-\pi / 2\right|^{\alpha+\beta} d \varphi_{1} \\
\leqq & c \rho^{-\alpha-\beta-1},
\end{aligned}
$$

where at the last step we have used $\beta+1<N$. (As pointed out in the introduction, the hypothesis of the theorem can only be satisfied if $\beta<1$ and we have $N \geqq 2$. 
Even apart from this, the best one could hope for in (3.13) is to get $\beta=N-1$.) The next step is to investigate the functions $h_{n}\left(\varphi_{1}\right)$. When $\xi$ and $\eta$ are given by (3.5) and (3.6), then

$$
(\xi-\eta, \theta)=B_{1}(\theta) \cos \varphi_{1}+B_{2}(\theta)\left(\sin \varphi_{1}-1\right),
$$

where the dependence of $B_{2}$ on $\varphi_{2}, \ldots, \varphi_{N-1}$ has been suppressed, but $B_{2}$ is bounded. Thus, for all $n, h_{n}\left(\varphi_{1}\right)$ is a polynomial in the two variables $\sin \varphi_{1}, \cos \varphi_{1}$. The same must then be true for $\left\{\sum_{n=1}^{k-1} h_{n}\left(\varphi_{1}\right)\right\}^{j}$. It follows that Lemma 4 may be applied to show that $I_{4}(\rho)=O\left(\rho^{-\alpha-N}\right)$.

Combining this with (3.9)-(3.11) and (3.13) completes the proof except for a comment on the situation when $N=2$ and the case of $\alpha=1$. When $N=2$, the range of integration for $\varphi_{1}$ is $[0,2 \pi]$ and we have only discussed $[0, \pi]$. However, it is clear that the integral over $[\pi, 2 \pi]$ can be split up in the same way with $h(3 \pi / 2)$ playing the role of $h(\pi / 2)$.

We now sketch briefly the argument for $\alpha=1$. Consider

$$
\begin{aligned}
& I(\rho)+I(-\rho)= \int_{0}^{\infty} \int_{0}^{\pi} \exp \left(-\operatorname{ir} \rho \cos \varphi_{1}-r h\left(\varphi_{1}\right)\right) r^{N-1}\left(\sin \varphi_{1}\right)^{N-2} d \varphi_{1} d r \\
&+\int_{0}^{\infty} \int_{0}^{\pi} \exp \left(\operatorname{ir} \rho \cos \varphi_{1}-r h\left(\varphi_{1}\right)\right) r^{N-1}\left(\sin \varphi_{1}\right)^{N-2} d \varphi_{1} d r \\
&= \int_{0}^{\infty} \int_{0}^{\pi} \exp \left(-\operatorname{ir} \rho \cos \varphi_{1}\right)\left\{\exp \left(-r h\left(\varphi_{1}\right)\right)+\exp \left(-r h\left(\pi-\varphi_{1}\right)\right)\right\} \\
& \times r^{N-1}\left(\sin \varphi_{1}\right)^{N-2} d \varphi_{1} d r
\end{aligned}
$$

the substitution $\varphi_{1} \rightarrow \pi-\varphi_{1}$ having been made in the second integral. Since $I(\rho) \leqq I(\rho)+I(-\rho)$, it will suffice to prove the last integral is $O\left(\rho^{-2-\beta}\right)$. Let

$$
\begin{aligned}
& A_{5}\left(r, \varphi_{1}\right)=\exp (-r h(\pi / 2))\left\{2 h(\pi / 2)-h\left(\varphi_{1}\right)-h\left(\pi-\varphi_{1}\right)\right\} r, \\
& A_{6}\left(r, \varphi_{1}\right)=2 \exp (-r h(\pi / 2)), \\
& A_{7}\left(r, \varphi_{1}\right)=\exp \left(-r h\left(\varphi_{1}\right)\right)+\exp \left(-r h\left(\pi-\varphi_{1}\right)\right)-A_{5}\left(r, \varphi_{1}\right)-A_{6}\left(r, \varphi_{1}\right),
\end{aligned}
$$

and define $I_{n}(\rho)$ by (3.8). Then

$$
I(\rho)+I(-\rho)=I_{5}(\rho)+I_{6}(\rho)+I_{7}(\rho),
$$

and $I_{6}(\rho)=O\left(\rho^{-1-N}\right)$ by Lemma 3 as before. Using $m=2$ in Lemma 5 and (3.4) with $\varepsilon<(1-\beta) / 2$ and splitting the $\varphi_{1}$-integration as we did with $I_{3}(\rho)$, we see that

$$
I_{7}(\rho)=O\left(\rho^{-3+2 \varepsilon}\right)=O\left(\rho^{-2-\beta}\right) .
$$

Finally, using the corollary which follows Lemma 9 together with Lemma 2, the estimate $I_{5}(\rho)=O\left(\rho^{-2-\beta}\right)$ is obtained in the same way as the estimate for

$$
I_{1}(\rho)-I_{4}(\rho) \text {. }
$$

REMARKS. In the proof of Theorem 1, note that good estimates were found for the integrals $I_{2}(\rho), I_{3}(\rho)$, and $I_{4}(\rho)$. The difficulty in improving the theorem in 
dimensions higher than two comes with the estimate for $I_{1}(\rho)-I_{4}(\rho)$. This required an expansion for $g$ and more terms in the expansion would be required in order to obtain better results from the present method of proof.

4. The potential kernel of a stable process. If $\alpha>(N-1) / 2$, the estimate of Theorem 1 shows that if $t$ is small, $|x|=1$, then

$$
p(t, x)=p\left(1, t^{-1 / \alpha} x\right) t^{-N / \alpha} \leqq K t^{1+(1-N) / \alpha}=K t^{c}
$$

with $c>-1$. This implies that $\int_{0}^{1} p(t, x) d t$ converges uniformly for $|x|=1$. Since the density is bounded, we also have $p(t, x) \leqq K t^{-N / \alpha}$ and since we are assuming $N>\alpha($ see $\S 2) \int_{1}^{\infty} p(t, x) d t$ converges uniformly for $|x|=1$. The argument given in [12] is therefore valid in this case so that

$$
U(x) \leqq K|x|^{\alpha-N}
$$

for any stable process in $R^{N}$ with index $\alpha>(N-1) / 2$.

We now go on to consider the case $\alpha \leqq(N-1) / 2$. In order to show that the integral of the potential kernel is not too badly behaved (even though the kernel itself may be infinite on some rays) we will need to show that the average behaviour of the stable density is the same as in the symmetric case so that on average it decays like $|x|^{-N-\alpha}$ even though the decay may be slower in some directions. It is convenient to have this result in the following slightly different form.

THEOREM 2. Let $p(1, x)$ be a stable density of index $\alpha$ in $R^{N}$ and let $D(\rho, h)$ denote a spherical shell:

$$
D(\rho, h)=\left\{x \in R^{N}: \rho \leqq|x| \leqq \rho+h\right\} .
$$

Then there is a constant $K$ such that

$$
\int_{D(\rho, h)} p(1, x) d x \leqq K \rho^{-1-\alpha} h
$$

Proof. By the inversion formula

$$
\int_{D(\rho, h)} p(1, x) d x=(2 \pi)^{-N} \int_{R^{N}} \int_{D(\rho, h)} \exp \left(-i(x, \xi)-|\xi|^{\alpha} g(\xi)\right) d x d \xi
$$

Now for each fixed $\xi$ we make an orthogonal transformation on $x$ given by $\eta=B x$ with $b_{1 j}=\xi_{j} /|\xi|, j=1,2, \ldots, N$. Then

$$
(x, \xi)=\sum_{j=1}^{N} x_{j} \xi_{j}=|\xi| \sum_{j=1}^{N} b_{1 j} x_{j}=|\xi| \eta_{1}
$$

and since the orthogonal transformation has Jacobian \pm 1 and preserves $D(\rho, h)$ we have

$$
\int_{D(\rho, h)} p(1, x) d x=(2 \pi)^{-N} \int_{R^{N}} \int_{D(\rho, h)} \exp \left(-i \eta_{1}|\xi|-|\xi|^{\alpha} g(\xi)\right) d \eta d \xi
$$


Next change $\eta$ to spherical coordinates:

$$
\begin{aligned}
\int_{D(\rho, h)} p(1, x) d x= & (2 \pi)^{-N} \int_{R^{N}} \int_{0}^{\rho+h} \int_{0}^{2 \pi} \cdots \int_{0}^{\pi} \exp \left(-i r \cos \varphi_{1}|\xi|-|\xi|^{\alpha} g(\xi)\right) \\
& \cdot J\left(r, \varphi_{1}, \ldots, \varphi_{N-1}\right) d \varphi_{1} \ldots d \varphi_{N-1} d r d \xi \\
= & c \int_{R^{N}} \int_{0}^{\rho+h} \int_{0}^{2 \pi} \cdots \int_{0}^{\pi} J_{(N-2) / 2}(r|\xi|)(r|\xi|)^{-(N-2) / 2} \exp \left(-|\xi|^{\alpha} g(\xi)\right) \\
& \cdot r^{N-1}\left(\sin \varphi_{2}\right)^{N-3} \ldots \sin \varphi_{N-2} d \varphi_{2} \ldots d \varphi_{N-1} d r d \xi \\
= & c^{\prime} \int_{R^{N}} \int_{0}^{\rho+h} J_{(N-2) / 2}(r|\xi|) \exp \left(-|\xi|^{\alpha} g(\xi)\right)|\xi|^{-(N-2) / 2} r^{N / 2} d r d \xi
\end{aligned}
$$

The next step is to change $\xi$ to spherical coordinates and perform the integration with respect to $|\xi|$. The Jacobian contributes a factor of $|\xi|^{N-1}$. Recalling that $g(\xi)$ is independent of $|\xi|$ and applying Lemma 1, we see that this integration produces an integrand of order $r^{-\alpha-1-N / 2} r^{N / 2}$ times the angular components of the Jacobian from the change of $\xi$ to spherical coordinates. After integrating the angular components of $\xi$, we have

$$
\int_{D(\rho, h)} p(1, x) d x \leqq K \int_{\rho}^{\rho+h} r^{-\alpha-1} d r \leqq K \rho^{-\alpha-1} h .
$$

If $\left(r, \varphi_{1}, \ldots, \varphi_{N-1}\right)$ denotes the representation of a point in $R^{N}$ in spherical coordinates, then restricting the angles $\varphi_{t}$ by

$$
\left|\varphi_{i}-\psi_{i}\right|<\delta, \quad i=1,2, \ldots, N-1,
$$

where $\delta>0$ and $\psi_{1}, \ldots, \psi_{N-1}$ are fixed, gives a type of cone in $R^{N}$ with vertex at the origin. Let

$$
D(\rho, h, \delta)=\left\{\left(r, \varphi_{1}, \ldots, \varphi_{N-1}\right): \rho \leqq r \leqq \rho+h,\left|\varphi_{i}-\psi_{i}\right|<\delta, i=1, \ldots, N-1\right\},
$$

the intersection of such a cone with the shell $D(\rho, h)$. The remainder of this section will be devoted to the application of Theorems 1 and 2 to the estimation of the integral of the potential kernel $U(x)$ (see (2.3)) over the set $D(\rho, h, \delta)$.

The first step is to use a change of scale to convert the estimate in Theorem 2 into one involving $t$ :

$$
\begin{aligned}
\int_{D(\rho, h)} p(t, x) d x & =\int_{D(\rho, h)} p\left(1, t^{-1 / \alpha} x\right) t^{-N / \alpha} d x=\int_{D\left(t^{-1 / \alpha} \rho, t^{-1 / \alpha} h\right)} p(1, \eta) d \eta \\
& \leqq K \rho^{-1-\alpha} h t .
\end{aligned}
$$

Here we have used the change of variable $\eta=t^{-1 / \alpha} x$. The volume of $D(\rho, h, \delta)$ is proportional to $\rho^{N-1} \delta^{N-1} h$ so the upper bound on the density given by Theorem 1 (with $\beta=0$ ) leads to

$$
\int_{D(\rho, h, \delta)} p(1, x) d x \leqq c \rho^{N-\alpha-2} \delta^{N-1} h
$$


Using the change of scale as before, this yields

$$
\int_{D(\rho, h, \delta)} p(t, x) d x \leqq c \rho^{N-\alpha-2} \delta^{N-1} h t^{1-(N-1) / \alpha} .
$$

Now let us work on the kernel; under the assumption $\alpha<(N-1) / 2$,

$$
\begin{aligned}
\int_{D(\rho, h, \delta)} U(x) d x & =\int_{0}^{\infty} d t \int_{D(\rho, h, \delta)} p(t, x) d x \\
& \leqq \int_{0}^{(\rho \delta)^{\alpha}} K \rho^{-1-\alpha} h t d t+\int_{(\rho \delta)^{\alpha}}^{\infty} c \rho^{N-\alpha-2} \delta^{N-1} h t^{1-(N-1) / \alpha} d t
\end{aligned}
$$

using (4.2), (4.3), and $D(\rho, h, \delta) \subset D(\rho, h)$. Performing the integration,

$$
\int_{D(\rho, h, \delta)} U(x) d x \leqq c^{\prime} \rho^{-1+\alpha} h \delta^{2 \alpha} .
$$

For the case $\alpha=(N-1) / 2$, we need one more estimate which comes from the fact that the density is bounded

Now if $\delta \leqq 1$,

$$
\begin{aligned}
\int_{D(\rho, h, \delta)} p(t, x) d x & =\int_{D(\rho, h, \delta)} p\left(1, t^{-1 / \alpha} x\right) t^{-N / \alpha} d x \\
& \leqq c_{1} t^{-N / \alpha} \rho^{N-1} \delta^{N-1} h .
\end{aligned}
$$

$$
\begin{aligned}
\int_{D(\rho, h, \delta)} U(x) d x \leqq \int_{0}^{(\rho \delta)^{\alpha}} K \rho^{-1-\alpha} h t d t & +\int_{(\rho \delta)^{\alpha}}^{\rho^{\alpha}} c \rho^{N-\alpha-2} \delta^{N-1} h t^{1-(N-1) / \alpha} d t \\
& +\int_{\rho^{\alpha}}^{\infty} c_{1} \rho^{N-1} \delta^{N-1} h t^{-N / \alpha} d t .
\end{aligned}
$$

Evaluating the integrals and remembering that $\alpha=(N-1) / 2$ gives

$$
\int_{D(\rho, h, \delta)} U(x) d x \leqq c_{2} \rho^{-1+\alpha} h \delta^{2 \alpha}\left(1+c_{3}|\log \delta|\right) .
$$

If $\delta>1$, the upper limit in the first integral can be changed to $\rho^{\alpha}$ and the middle integral ignored with the result that $(4.5)$ is still valid.

5. Hitting probabilities. We start with an interpretation of the potential generated by Lebesgue measure on a set.

LEMMA 10. If $F$ is a set of positive Lebesgue measure, and $T_{F}$ denotes the total time spent in $F$ by the process, then

Proof.

$$
E^{x}\left\{T_{F}\right\}=\int_{F} U(y-x) d y .
$$

$$
\begin{aligned}
E^{x}\left\{T_{F}\right\} & =\int_{0}^{\infty} P^{x}[X(t) \in F] d t \\
& =\int_{0}^{\infty} d t \int_{F} p(t, y-x) d y \\
& =\int_{F} U(y-x) d y .
\end{aligned}
$$


Now if $\tau$ is any stopping time, since shifting the path can only reduce $T_{F}$, the strong Markov property gives

$$
E^{x}\left\{T_{F}\right\} \geqq E^{x} E^{X(\tau)}\left\{T_{F}\right\} .
$$

We will apply this when $\tau$ is the hitting time of a set $E$, i.e.

$$
\tau_{E}=\inf \{t>0: X(t) \in E\} .
$$

If $E$ is closed, then $X\left(\tau_{E}\right) \in E$, so if we put $\lambda=\inf _{x \in E} E^{x}\left\{T_{F}\right\}$, then we will have

$$
E^{x}\left\{T_{F}\right\} \geqq \lambda \Phi(x, E)
$$

where $\Phi(x, E)$ denotes the probability of hitting $E$, starting at $x$. This will now be used to obtain bounds for $\Phi(x, E)$ when $E$ is a sphere.

THEOREM 3. Suppose $E$ is a sphere of radius $r$ and $x$ is at a distance $d$ from $E$ with $r \leqq d$. For a fixed stable process of index $\alpha<N$ in $R^{N}$ (restricted to satisfy (2.2) if $\alpha=1)$, there is a constant $c$ such that

(i) if $\alpha>(N-1) / 2, \Phi(x, E) \leqq c(r / d)^{N-\alpha}$;

(ii) if $\alpha=(N-1) / 2, \quad \Phi(x, E) \leqq c(r / d)^{N-\alpha}(1+\log d / r)$;

(iii) if $\alpha<(N-1) / 2, \quad \Phi(x, E) \leqq c(r / d)^{1+\alpha}$.

If the process is of type $A$, there is a constant $K$ with $\Phi(x, E) \geqq K(r / d)^{N-\alpha}$.

REMARK. The estimates in (ii) and (iii) can be improved if we know that the measure $\mu$ appearing in the characteristic function of the stable process satisfies the condition of Theorem 1 for some $\beta>0$. We do not state the general result as it seems far from best possible; note, however, that if

$$
\int_{S_{N}}|(\xi, \theta)|^{-\beta} \mu(d \theta) \leqq M
$$

for some $\beta>N-1-2 \alpha$, then we have $\Phi(x, E) \leqq c(r / d)^{N-\alpha}$, so that in this case the hitting probability of a sphere has the same order of magnitude as for the corresponding symmetric process. The example considered in the next section shows that the estimates (ii) and (iii) cannot be improved in general.

Proof. The lower bound for processes of type $A$ was obtained in [12]. If $\alpha>(N-1) / 2$, it is a consequence of Theorem 1 , with $\beta=0$, that the arguments in [12] are valid for the upper bound also as we have observed at the beginning of $\$ 4$. We proceed to prove (ii) and (iii). First note that we can assume that $d \geqq 2 r$ since these estimates are trivially true otherwise. The total time spent in a sphere of radius $r$ by a process starting at its centre has expectation $K r^{\alpha}$ by the scaling property (2.1). If we take for $F$ the sphere of radius $2 r$, concentric with $E$, then the sphere with centre at $x$, radius $r$ will be contained in $F$ for every $x \in E$. Thus $\lambda=\inf _{x \in E} E^{x}\left\{T_{F}\right\} \geqq K r^{\alpha}$, so that by (5.1) and Lemma 10 ,

$$
\Phi(x, E) \leqq K^{-1} r^{-\alpha} E^{x}\left\{T_{F}\right\}=K^{-1} r^{-\alpha} \int_{F} U(y-x) d y .
$$


To estimate the last integral, let $D(\rho, h, \delta)$ be a sector which contains $F-x$; it suffices to let $\rho=d-r, h=4 r$, and $\delta=2 r \rho^{-1}$. Applying (4.4) in case (iii) and (4.5) in case (ii) completes the proof since in (5.2) we have

$$
\int_{F} U(y-x) d y \leqq \int_{D(\rho, h, \delta)} U(z) d z .
$$

The assumption $d \geqq 2 r$ lets us replace $d-r$ by $d / 2$ at the last step.

6. Example. Suppose $X^{N}(t)=\left(X_{1}^{N}(t), X_{2}^{N}(t), \ldots, X_{N}^{N}(t)\right)$ denotes the stable process in $R^{N}$ obtained by assuming that the coordinate processes $X_{i}^{N}(t)$ are independent symmetric stable processes of index $\alpha$ in $R^{1}$. This corresponds to a measure $\mu$ on $S_{N}$ which consists of $2 N$ equal point masses at the points where the coordinate axes intersect $S_{N}$. We could also think of $X^{N}(t)$ as the sum of $N$ independent linear processes in different directions. It is clear that the density $p(t, x)$ for this process is of the form $p(t, x)=p_{1}\left(t, x_{1}\right) p_{1}\left(t, x_{2}\right) \ldots p_{1}\left(t, x_{N}\right)$ where $x=\left(x_{1}, x_{2}, \ldots, x_{N}\right)$ and $p_{1}\left(t, x_{1}\right)$ is the density for a linear symmetric stable process of index $\alpha$. Since [11]

$$
\begin{array}{ll}
c_{1} \leqq p_{1}(1, x) \leqq c_{2} & \text { for }|x| \leqq 1, \\
c_{3} \leqq|x|^{1+\alpha} p_{1}(1, x) \leqq c_{4} & \text { for }|x| \geqq 1,
\end{array}
$$

it is easy to check that $U(x)=\int_{0}^{\infty} p(t, x) d t$ will be finite for $x \neq 0$ if $\alpha>(N-1) / 2$, but will be infinite for $x$ on a coordinate axis if $\alpha \leqq(N-1) / 2$. (The scaling property is used in this computation much as it was in (4.2), for example.) We now estimate $U(x)$ for points close to one of the axes. Consider the set

$$
C=\left\{x: \rho \leqq x_{1} \leqq \rho+h,\left|x_{i}\right| \leqq a, i=2, \ldots, N\right\}
$$

for $a$ and $h$ small compared to $\rho$, say $\max \{2 a, h\} \leqq \rho$. Then $C$ will differ very little from $D(\rho, h, \delta)$ with $\delta=a \rho^{-1}$. For $x \in C$,

$$
\begin{aligned}
U(x) & =\int_{0}^{\infty} p(t, x) d t \geqq \int_{a^{\alpha}}^{\rho^{\alpha}} \prod_{j=1}^{N} p_{1}\left(1, t^{-1 / \alpha} x_{j}\right) t^{-N / \alpha} d t \\
& \geqq c_{3} c_{1}^{N-1} x_{1}^{-1-\alpha} \int_{a^{\alpha}}^{\rho^{\alpha}}\left(t^{-1 / \alpha}\right)^{-1-\alpha} t^{-N / \alpha} d t
\end{aligned}
$$

by (6.1), since $t^{-1 / \alpha} x_{1} \geqq 1$ for $t \leqq \rho^{\alpha}$ and $t^{-1 / \alpha}\left|x_{j}\right| \leqq 1$ for $t \geqq a^{\alpha}$. Performing the integration, we obtain

$$
\begin{array}{ll}
U(x) \geqq K_{1} \rho^{-1-\alpha} a^{2 \alpha-N+1}, & \text { if } \alpha<(N-1) / 2, \\
U(x) \geqq K_{2} \rho^{-1-\alpha} \log \rho / a, & \text { if } \alpha=(N-1) / 2 .
\end{array}
$$

These estimates could be used to show that (4.4) and (4.5) are best possible in general, but we will procede directly to the estimation of the hitting probabilities. Recall that

$$
\Phi(x, E)=\int_{E} U(y-x) \nu(d y)
$$


where $v$ is the capacitory measure for the compact set $E$. If $E$ is the sphere of radius $r$ centred at the point $(d+r, 0, \ldots, 0)$, then $E \subset C$ with $\rho=d, h=2 r, a=r$. Thus, if $\alpha<(N-1) / 2$ and $d \geqq 2 r$, it follows from (6.2) and the fact (Lemma 3 of [12]) that the capacity of a sphere of radius $r$ is $K_{3} r^{N-\alpha}$ that

$$
\begin{aligned}
\Phi(0, E) & \geqq K_{1} d^{-1-\alpha} r^{2 \alpha-N+1} v(E) \\
& =K_{1} K_{3} d^{-1-\alpha} r^{\alpha+1} .
\end{aligned}
$$

Similarly, if $\alpha=(N-1) / 2$ and $d \geqq 2 r$,

$$
\Phi(0, E) \geqq K_{2} K_{3} d^{-1-\alpha} r^{\alpha+1} \log (d / r) .
$$

These results show that the estimates in Theorem 3 (ii) and (iii) for $\Phi(x, E)$ are of the right order of magnitude.

Further examination of this example shows that while the hitting probabilities of spheres located in certain directions from the starting point can be larger than for the symmetric stable process of the same index as we have seen, in other directions these probabilities will be of the same order of magnitude as they are in the symmetric case. Thus, for the process we are considering, completely different orders of magnitude are possible for the hitting probabilities of spheres located in different directions. Intuitively this is explained by the possibility of a large jump in the preferred direction in a small time before the other components of the process have had a chance to grow. This intuitive idea becomes precise in the next section.

7. Delayed hitting probabilities. The purpose of this section is to state for purposes of comparison the result that if we consider the probability of hitting the sphere after a fixed positive time $T$ (and if the starting point is not too far away) then the "delayed hitting probability" will be of the same order of magnitude for all stable processes of a given index. Let

$$
Q(x, E, T)=P^{x}[X(t) \in E \text { for some } t \geqq T] .
$$

Then we have

THEOREM 4. Suppose $E$ is a sphere of radius $r$. For a fixed stable process of index $\alpha<N$ in $R^{N}$ (restricted to satisfy (2.2) if $\alpha=1$ ), there is a constant $c$ such that

$$
Q(x, E, T) \leqq c\left(r / T^{1 / \alpha}\right)^{N-\alpha} .
$$

If the process is of type $A, x \in E$, and $r \leqq T^{1 / \alpha}$, then there is a constant $K$ such that

$$
Q(x, E, T) \geqq K\left(r / T^{1 / \alpha}\right)^{N-\alpha} .
$$

REMARK. The upper bound shows that for a sphere of radius $r$ at a distance $d$, the probability of hitting this sphere after time $d^{\alpha}$ is no larger than $c(r / d)^{N-\alpha}$, as it is in the symmetric case. Thus, as we mentioned earlier, the large estimates in (ii) and (iii) of Theorem 3 must be due to large excursions in specific directions in 
relatively small times. (Note that the expected length of time to first travel the distance $d$ is of order $d^{\alpha}$.)

Proof. The upper bound is Lemma 2.2 of [4]. From the proof of that lemma, we take the equality

$$
Q(x, E, T)=\int_{E} \int_{T}^{\infty} p\left(1, s^{-1 / \alpha}(z-x)\right) s^{-N / \alpha} d s \nu(d z)
$$

where $\nu$ is the capacitory measure for $E$. (There is a misprint in [4], the exponent for $s$ there reading $N-\alpha$. However, (7.1) is the correct version.) Now $\left|s^{-1 / \alpha}(z-x)\right|$ $\leqq T^{-1 / \alpha} 2 r \leqq 2$ and since the process is type $A$, the density is bounded below on the sphere of radius 2 , centre the origin. Thus

$$
Q(x, E, T) \geqq K_{1} v(E) \int_{T}^{\infty} s^{-N / \alpha} d s
$$

Performing the integration and recalling that $\nu(E)=K_{2} r^{N-\alpha}$ (Lemma 3 of [12]) completes the proof.

As we have pointed out in the introduction, Theorem 4 is sufficient to justify Theorems 2 and 3 of [12].

8. Further problems. The problem of improving the hypothesis of Theorem 1 (particularly for $N \geqq 3$ ) has already been mentioned in the introduction. In addition, it would seem desirable to know whether it is possible to obtain the more complete information about the asymptotic behaviour of the stable density that is available in one dimension [11].

There are some interesting problems concerning polar sets. For $\alpha=1, N=1$, it is known that the nonsymmetric Cauchy processes have no nonempty polar sets [9], while the symmetric process has nonempty polar sets. If $N \geqq 2$, does the class of polar sets for $\alpha=1$ vary with the process? It seems likely that (as in $R^{1}$ ) it may differ at least for processes not having the scaling property. A similar problem now exists for general $\alpha$. Orey [6] showed that all stable processes of index $\alpha$ in $R^{N}$ which satisfy

$$
U(x) \leqq c|x|^{\alpha-N}
$$

have the same polar sets. However, we have seen that $(8.1)$ is false in general. It remains an open question, therefore, whether the class of polar sets is the same for all stable processes with given $N \geqq 2, \alpha \neq 1$. For $N=1$, there is no problem since (8.1) is then satisfied for $\alpha<1$.

Another question we have ignored entirely is the behaviour of processes with index $\alpha=1$ not satisfying the scaling property. For example, in this case we have not even considered the asymptotic behaviour of the density. Of course, for $N=1$ this is given in [11]. Also the asymptotic behaviour of $U$ in this case is obtained in [9]. 


\section{REFERENCES}

1. R. M. Blumenthal and R. K. Getoor, Some theorems on stable processes, Trans. Amer. Math. Soc. 95 (1960), 263-273.

2. A. Erdélyi et al., Higher transcendental functions, Vol. II, Bateman Manuscript Project, McGraw-Hill, New York, 1953.

3. G. A. Hunt, Markov processes and potentials. I, II, III, Illinois J. Math. 1 (1957), 44-93, 316-369; 2 (1958), 151-213.

4. N. Jain and W. E. Pruitt, The correct measure function for the graph of a transient stable process, Z. Wahrscheinlichkeitstheorie und Verw. Gebiete 9 (1968), 131-138.

5. P. Lévy, Théorie de l'addition des variables aléatoires, Gauthier-Villars, Paris, 1937.

6. S. Orey, "Polar sets for processes with stationary independent increments" in Markov processes and potential theory, edited by J. Chover, Wiley, New York, 1967, pp. 117-126.

7. G. Polya, On the zeroes of an integral function represented by Fourier's integral, Messenger of Math. 52 (1923), 185-188.

8. S. C. Port, A remark on hitting places for transient stable process, Ann. Math. Statist. 39 (1968), 365-371.

9. S. C. Port and C. J. Stone, The asymmetric Cauchy processes on the line, Ann. Math. Statist. 40 (1969), 137-143.

10. W. E. Pruitt and S. J. Taylor, Sample path properties of processes with stable components, Z. Wahrscheinlichkeitstheorie und Verw. Gebiete 12 (1969), 267-289.

11. A. V. Skorohod, Asymptotic formulas for stable distribution laws, Dokl. Akad. Nauk SSSR 98 (1954), 731-734; English transl., Selected Transls. in Math. Stat. and Prob. 1 (1961), 157-161.

12. S. J. Taylor, Sample path properties of a transient stable process, J. Math. Mech. 16 (1967), 1229-1246.

13. G. N. Watson, A treatise on the theory of Bessel functions, Cambridge Univ. Press, Cambridge, 1944.

UNIVERSITY OF MINNESOTA, MiNNEAPOLIS, MinNESOTA

WestField COllege,

LONDON, ENGLAND 